\title{
Can restaurant revenue management work with menu analysis?
}

\begin{abstract}
Menu analysis and revenue management approaches contribute to improving a restaurant's profitability. Yet, both approaches are often implemented independently with constraints. This paper explores the potential of integrating both approaches to improve strategy formulation. Hence, this paper identified the extent of applicability and synergies among both approaches. Findings highlighted that as both approaches become more sophisticated, the practicality of implementation will deprive. The synergies identified the potential to integrate both approaches' performance indicators, cost efficiency data and strategies. Understanding the applicability and synergies of both approaches will lay the foundation for an effective integrated menu analysis and revenue management framework.
\end{abstract}

Keyword: Restaurant revenue management; Menu analysis; Menu management; Profitability management; Performance management; Cost control 